\title{
PLANEACIÓN PROSPECTIVA Y ESTRATÉGICA EN EDUCACIÓN DESDE UN ENTORNO DE MERCADO. LA EXPERIENCIA DEL PROGRAMA TECNOLOGÍA DE AUDIO DE LA UNIVERSIDAD NACIONAL ABIERTA Y A DISTANCIA, UNAD
}

\author{
Juan Pablo Rodríguez Guevara'
}

\section{Resumen}

En el presente artículo se expone el caso de la Tecnología de Audio de la Universidad Nacional Abierta y a Distancia UNAD, donde se llevó a cabo el proceso de diagnóstico y de planeación estratégica del programa, en el marco de desarrollo de un estudio que tenía como finalidad la construcción de un Plan Estratégico de Mercadeo Online para la promoción de dicha oferta académica. El enfoque de planeación estratégica que se tomó en el trabajo, se centra en la planeación como una actividad que permite reforzar estructuras y procesos existentes en la Tecnología de Audio, con el fin de promover el mejoramiento, crecimiento y la permanencia a lo largo del tiempo de la misma, siguiendo dos de las estrategias genéricas planteadas por Michael Porter; la de diferenciación y la de enfoque o alta segmentación. El desarrollo del trabajo se llevo a cabo en tres etapas básicas que enmarcaron el desglose general del mismo. En la primera de ellas se dio origen al diagnóstico y a la definición de los objetivos estratégicos del programa, a través del modelo de planeación estratégica que tiene como componentes fundamentales, el direccionamiento, el diagnóstico, la formulación estratégica y finalmente la evaluación estratégica (Amaya, 2005). En la segunda etapa se desarrolló la investigación de mercado y los planteamientos de las diferentes estrategias de mercadeo, haciendo énfasis en tres de los cuatro componentes de la mezcla de mercado; la plaza, el servicio y la promoción. Finalmente en la última etapa se construyó la propuesta para la implementación operativa y la evaluación de la iniciativa de mercadeo. En este artículo se abordarán en profundidad los procesos de análisis, metodologías aplicadas y los resultados alcanzados en las dos primeras etapas del proyecto, puesto que fue aquí donde precisamente surgieron los aportes más significativos...

Palabras claves: Planeación, educación, estrategia, mercadeo, diagnóstico, promoción.

1 Ingeniero de Sonido y Administrador de Empresas con especialización en Pedagogías para el Desarrollo del Aprendizaje Autónomo. Docente del programa de Tecnología de Audio de la Universidad Nacional Abierta y a 


\title{
PROSPECTS AND STRATEGIC PLANNING IN EDUCATION FROM A MARKET ENVIRONMENT - THE EXPERIENCE PROGRAM AUDIO TECHNOLOGY NA- TIONAL COLLEGE OPEN AND DISTANCE UNAD
}

\begin{abstract}
In the next article we are going to show the case of audio technology of Universidad Nacional Abierta y a Distancia UNAD, where the diagnostic and strategic planning process of the program were set, this was made under the development of a studio which had as goals the construction of an online marketing strategic plan to promote this academic offer. The development of the work was made in three basic stages. In the first stage the diagnostic and the definition of strategic objectives of the program had their origin; in the second stage the marketing research and the the different marketing planning strategies were developed. Finally in the las stage a proposal for the operative application of the marketing strategy was built. In this article we are going to take a deep look into analyze process, apply methodology. And the achieve results in the first and second stages of the project, because the most significant ideas came out in those stages.
\end{abstract}

Keywords: Planning, education, strategy, marketing, diagnosis, promotion.

\section{Introducción}

Las organizaciones educativas al igual que las empresas requieren indispensablemente de la implementación de diversos procesos y acciones administrativas para gestionar adecuadamente sus recursos, garantizar el buen funcionamiento y procurar la permanencia a lo largo del tiempo de las mismas.

Los programas de pregrado de orden profesional, técnico y tecnológico que se ofrecen en las universidades, no solo necesitan de los contenidos y sistemas académicos que se consolidan como la columna vertebral de la oferta educativa, sino que también precisan de la gestión como vehículo moderador y cohesionador que le da sentido, orden, rumbo y refuerza la estructura general del programa académico.

Cuando se habla de la gestión de un programa de formación académica como mecanismo de apoyo, moderación y cohesión, se hace referencia a los procesos administrativos que cumplen las tareas de planear, organizar, dirigir, coordinar y controlar los elementos y recursos humanos, físicos, financieros, logísticos, sociales, académicos y de mercado (Fayol,1916), que se articulan en torno a la estructura organizacional del programa, buscando optimizar en términos de calidad, eficiencia y eficacia, las intencionalidades de formación que persigue la oferta educativa de pregrado.

En este sentido, el presente artículo pretende dar a conocer el trabajo que se llevó a cabo en el programa de Tecnología de Audio de la Universidad Nacional Abierta y a Distancia UNAD, donde se realizó un estudio que tenía como finalidad el planteamiento de estrategias para potencializar los procesos de mercadeo del programa, partiendo del análisis interno y externo del mismo, con el fin de caracterizar la oferta educativa y plantar los cimientos para su planeación estratégica, determinando las fortalezas, debilidades, oportunidades y amenazas 
que tiene el programa en el actual entorno académico y de mercado.

Los resultados obtenidos tras el desarrollo del proyecto permitieron fortalecer el proceso de Autoevaluación del programa de Tecnología de Audio para el periodo 2008-2011, agregando elementos conceptuales y analíticos necesarios para la planeación y la construcción del plan de mejoramiento proyectado para su cumplimiento hasta el año 2015.

De igual forma, también soportaron el proceso de reestructuración de la Tecnología de Audio determinando los lineamientos sobre los cuales se realizó el cambio de denominación, el ajuste curricular, de créditos y de intencionalidad formativa del programa.

Por otra parte el estudio también contribuyó en el trabajo de creación del programa de Ingeniería de Audio de la UNAD, agregando elementos cuantitativos y cualitativos que enmarcan el escenario sobre el cual se diseñó el mismo, determinando algunas de las variables que se tuvieron en cuenta a la hora de definir la intencionalidad formativa del programa y sus contenidos curriculares. También permitió comprender las características y la dinámica del entorno académico y de mercado en el cual se encuentra la Ingeniería de Audio, señalando las principales oportunidades y amenazas con las que se enfrentará más adelante.

En cuanto a las estrategias de mercadeo se logró caracterizar la oferta educativa determinando los aspectos más importantes en relación al producto, el precio, la plaza y la promoción, confrontando los resultados encontrados con el ciclo de vida del producto, para después plantear las estrategias de mercadeo online y tradicional que buscaban incentivar el ingreso de nuevos estudiantes a la Tecnología de Audio.
Entre los aportes generados hasta el momento, se destaca la identificación y caracterización del segmento de mercado potencial para el programa, al igual que la ampliación la demanda y la visivilización del mismo tras la implementación de algunos de los elementos que se contemplan en el plan de mercadeo propuesto, como la apertura de espacios y la publicación de anuncios sobre redes sociales, al igual que la participación en ferias y eventos académicos como Campus Party.

\section{Diagnóstico del Programa Tecnología de Audio.}

La fase inicial del proyecto fue la base estructural que permitió realizar el desarrollo teórico y analítico que dio como resultado la determinación del diagnóstico general de la Tecnología, siguiendo el modelo de planeación estratégica integrado por cuatro componentes fundamentales que son el direccionamiento, el diagnostico, la formulación estratégica y la evaluación estratégica (Amaya, 2005). El establecimientos de indicadores, características y factores de carácter administrativo, organizacional y académico en relación al programa y todos sus ámbitos de influencia, facilitaron la construcción de una imagen global sobre el estado actual del mismo.

En este orden de ideas, el diagnóstico de la Tecnología de Audio se llevó a cabo a través del desarrollo de tres actividades básicas:

1. La identificación de los propósitos actuales del programa - Direccionamiento.

2. El análisis de la situación actual del programa (ambientes interno y externo) Diagnóstico.

3. El diseño de la estrategia maestra del programa - Formulación Estratégica. 


\section{Análisis Interno y Externo de la Tecnología de Audio.}

Para dar cumplimiento a los tres aspectos antes mencionados inicialmente se recurrió a la investigación, revisión y análisis histórico de las fuentes documentales del programa. Como fuente secundaria de información se tomaron los datos obtenidos tras la aplicación de algunas entrevistas con los directivos, administrativos y el personal docente de la Tecnología de Audio.

Todo lo anterior con el fin de recrear el trasegar histórico evolutivo de la Tecnología de Audio, para así identificar el conjunto de hechos y aspectos más significativos que han enmarcado los cambios estructurales, académicos y administrativos de los que ha sido objeto a partir del año 2007 cuando el Ministerio de Educación Nacional otorgó el Registro Calificado al programa.

Entre los cambios identificados y que agregan elementos conceptuales y analíticos importantes para la formulación del diagnóstico general de la tecnología, se tienen su virtualización en 2008 y la modificación de su estructura curricular en 2009.

Ahora bien, la identificación de los propósitos del programa se realizó tras el análisis de algunos aspectos claves que se tiene en cuenta a la hora de caracterizar un producto o en este caso, un servicio como la oferta educativa específica de la Tecnología de Audio. El público objetivo, el portafolio de servicios, el mercado, la filosofía, las ventajas competitivas, comparativas y algunos indicadores cuantitativos.

Gráfica I. Número de Estudiantes de la Tecnología de Audio.

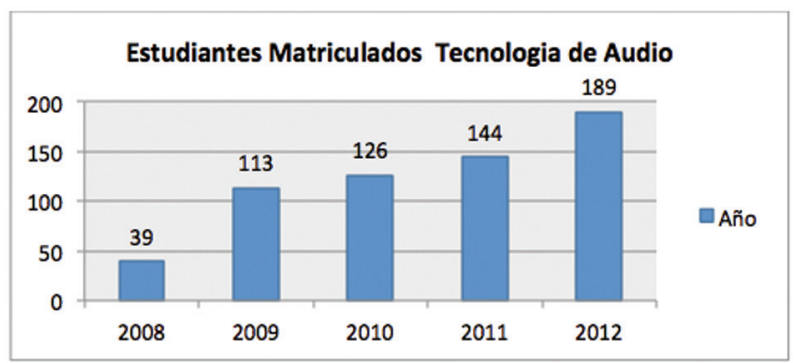

El resultado fue la construcción de un escenario base sobre el cual se llevó a cabo el análisis de la situación actual del programa. Establecer con claridad cuál es el propósito de la tecnología, es decir, el direccionamiento según el modelo de planeación estratégica propuesto por Amaya, fundamentado en la revisión y reflexión rigurosa de elementos como la visión, la misión y los objetivos organizaciones de la universidad y el programa en específico, permitió identificar el entramado organizacional e ideológico sobre la cual se deben plantear e implementar las estrategias que buscarán mejorar la estructura y los procesos que actualmente se tienen en la Tecnología de Audio. David Fred en su libro La Gestión Estratégica; al igual que muchos otros autores, advierten que el proceso de planeación estratégica se inicia y se fundamenta en la articulación de procesos, acciones y estrategias, que orbiten en torno a los lineamientos que plasman la identidad y la manera de hacer las cosas en una organización, como la misión, visión, los valores y las políticas organizacionales (Fred, 1989).

El segundo paso para el desarrollo del diagnóstico general del programa una vez establecido el punto de partida y la base analítica conceptual, fue una de las más importantes y relevantes puesto que en ella se determinaron las fortalezas, debilidades, amenazas y oportunidades con las cuales cuenta y a las que se debe enfrentar la Tecnología de Audio en el ejercicio práctico de sus funciones en medio de un entorno dinámico de mercado. Fred (1989) menciona que: «las estrategias deben aprovechar de forma efectiva las fortalezas de una firma, tratando de vencer sus debilidades, sacando mayor provecho de sus oportunidades externas claves y evitando las amenazas externas» (p.16).

Aquí se analizaron los ambientes interno y externo a través del establecimiento de factores claves que determinan el crecimiento y la permanencia a lo largo del tiempo de una oferta educativa. 
Ahora, el recurso metodológico que se empleó para relacionar, analizar y construir el diagnóstico y la estrategia maestra de la Tecnología de Audio; misión, visión y los objetivos estratégicos, fue el diseño de matrices de calificación y ponderación. La construcción de las mismas se inició con el establecimiento de grupos funcionales que describieron las diferentes sinergias que se generan entre los agentes que interactúan constantemente en el programa.

Gráfica II. Grupos Funcionales de Análisis.

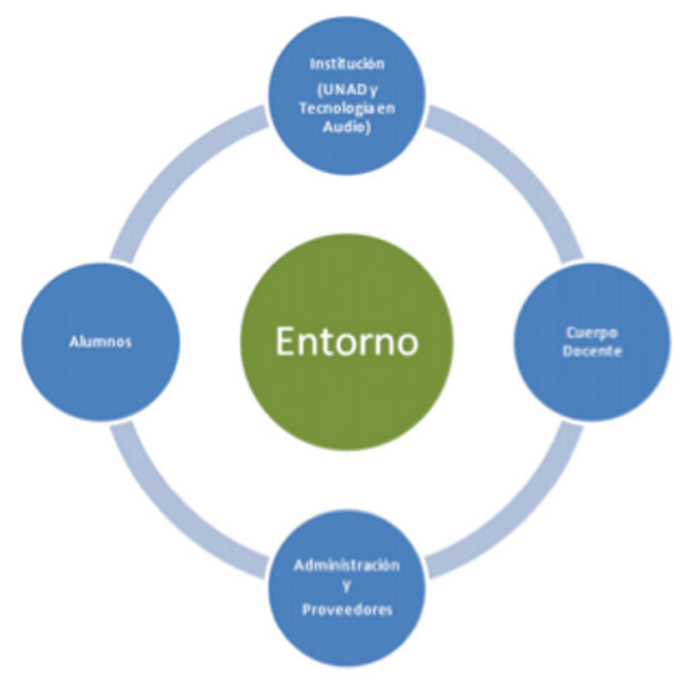

El resultado del ejercicio fue la definición de cuatro grupos funcionales con sus respectivos factores; profesores, estudiantes, personal administrativo, proveedores externos y la institución universitaria.

Una vez establecidos los factores por grupo funcional, se clasificaron los mismos para determinar cuáles eran los primarios estratégicos y cuáles los secundarios complementarios.

Esto se realizó examinando la recurrencia del factor en cada uno de los grupos funcionales relacionados anteriormente, un factor primario estratégico es aquel que tenía una frecuencia de tres y cuatro referencias en medio de los factores para la totalidad de los grupos; entre tanto, un factor secundario complementario se establecía cuando la frecuencia de las referencias es solo dos o uno.

El planteamiento anterior dio como resultado la identificación de diez factores primarios estratégicos y siete secundarios complementarios.

Después, se llevó a cabo la construcción de la matriz que sirvió como instrumento para analizar el estado actual del ambiente interno de la Tecnología de Audio. Con ella se lograron establecer las fortalezas y debilidades del programa.

En este sentido, la matriz se elaboró ponderando cada uno de los factores primarios y secundarios, con el fin de establecer el grado de importancia y de impacto que los mismos tenían en el desempeño, crecimiento y permanencia de la Tecnología de Audio a lo largo del tiempo. El grado de ponderación que se asignó para los factores de carácter estratégico fue del $70 \%$ mientras que para los secundarios complementarios fue del $30 \%$.

Una vez ponderados los factores, se calificaron los mismos en una escala de 1 a 10 donde el 1 significa la debilidad más importante y 10 la fortaleza más significativa que tenía el programa. Este procedimiento se realizó a través de la discusión y conciliación grupal del comité curricular de la tecnología, teniendo en cuenta documentos y material interno que facilitó el proceso.

Finalmente se multiplicó la calificación de cada factor por la ponderación asignada al mismo según la distribución porcentual de cada grupo; primarios y secundarios. La sumatoria de esos valores dio como resultado un indicador cuantitativo que ayudó a determinar si el programa académico era débil o fuerte. Como el resultado final posible oscilaba entre 1 y 10 y, además, su valor promedio era de 5.5 , se dedujo que la organización interna de la Tecnología de Audio tendía a ser relativamente débil ya que la suma 
total ponderada de todos los factores fue de 5.24 , valor que estaba por debajo del promedio de la escala de medición.

Después de todo el proceso antes mencionado, las fortalezas y debilidades identificadas fueron las siguientes:

Fortalezas: 6 de carácter estratégico y 3 de rigor secundario complementario.

1. Prestigio de la institución.

2. Aspectos económicos.

3. Tutoría.

4. Plataforma educativa.

5. Modalidad académica.

6. Administración.

7. Actualización tecnológica.

8. Desarrollo de contenidos y calidad de los mismos.

9. Planificación del programa.

Debilidades: 4 de carácter estratégico y 2 secundarias complementarias.

1. Capacitación docente.

2. Investigación.

3. Beneficio del programa educativo.

4. Infraestructura.

5. Tamaño del programa.

6. Demanda social del programa.

Continuando con el análisis y la construcción del diagnóstico general del programa, después de determinar las debilidades y fortalezas subyacentes a la estructura y el ambiente interno de la tecnología, se pasó al estudio de las fuerzas de mercado que determinan el escenario externo de interacción del programa. Con el análisis del ambiente externo se determinaron el conjunto de amenazas y oportunidades que tiene la Tecnología en Audio en el actual entorno de mercado.

En el estudio del ambiente externo se relacionaron y analizaron factores de carácter econó- mico, tecnológico, político, demográfico y social del país, al considerar los mismos como las fuerzas más influyentes en dicho contexto.

El resultado del ejercicio permitió definir los factores que influyen en el crecimiento, permanencia y la estructura organizacional de la Tecnología de Audio, en el marco de referencia planteado por el ambiente externo de la misma.

1. Ampliación de la cobertura de conectividad nacional (A). El crecimiento del número de conexiones a internet a lo largo de todo el territorio nacional facilitaría el suministro de la oferta educativa promovida por la UNAD.

2. Crecimiento y ampliación de espacios laborales relacionados con los campos de desempeño de los egresados de la Tecnología en Audio (B). La consolidación y ampliación del mercado laboral directamente relacionado con las áreas de desempeño de los egresados de la Tecnología en Audio brindaría mejores posibilidades al programa dada la pertinencia del mismo en el entorno social productivo del país.

3. Competencia con mejor calidad académica (C). La competencia en el mercado es un aspecto de gran importancia; los instrumentos con que se haga frente a dicha interacción, como la calidad académica, promoverán el crecimiento y la permanencia del programa Tecnología en Audio a lo largo del tiempo.

4. Desarrollo y adaptación a las nuevas metodologías de enseñanza que emplean las Tecnologías de la Información y las Comunicaciones (D). Las nuevas tecnologías de la información y las comunicaciones permitirán la optimización de los procesos de enseñanza y aprendizaje en la modalidad virtual, por tal motivo es importante su 
desarrollo y la capacidad institucional y humana para adaptarse adecuadamente a dichas tecnologías.

\section{Expansión de la demanda de programas} de formación tecnológica a nivel nacional e internacional (E). El crecimiento de la demanda nacional en relación a los programas de educación superior de carácter tecnológico, es un factor determinante para el crecimiento y la permanencia del programa sujeto de investigación.

6. Incremento de empresas en el sector del entretenimiento (F). La creación de empresas que generen productos o servicios que guarden algún tipo de relación con los enfoques de desempeño que tienen los Tecnólogos en Audio, generará al largo plazo la ampliación, crecimiento y consolidación de la demanda de dichos profesionales y del mercado específico en el cual se desenvuelven.

Ahora, siguiendo la misma metodología que se empleó en el análisis interno del programa, se generó una matriz que permitió formular los indicadores cuantitativos que llevaron a identificar el conjunto de amenazas y oportunidades que tiene la Tecnología de Audio en el actual entorno de académico y de mercado.

El resultado de todo el ejercicio de análisis antes mencionado, permitió inferir que las oportunidades y amenazas relacionadas con la Tecnología de Audio tienden a equilibrarse, porque tras su calificación y suma ponderada; 5.83 , se obtuvo un resultado que está por encima de la media 5.5. Para que un factor se consolide como una oportunidad para el programa su desarrollo futuro debe ser positivo, de lo contrario, si su progreso es negativo, el factor se concebirá como una amenaza para el programa.
La planeación estratégica y la definición de estrategias de acción en función de la diferenciación y el enfoque o alta segmentación, se fundamenta especialmente en la mirada objetiva de la situación actual del programa, puesto que a partir de ella, como se menciono anteriormente, se va a efectuar el proceso de planeación estratégica enfocado especialmente hacia el fortalecimiento de la estructura y los procesos existentes, con el fín de procurar el mantenimiento y crecimiento del programa en el actual entorno académico y de mercado. Las estrategias de diferenciación y de enfoque como su nombre lo indican, buscan esencialmente la incorporación en el servicio educativo, de características tangibles e intangibles que generen un mayor valor en la percepción de la demanda potencial del programa, al igual que responder puntualmente a las necesidades y expectativas del segmento de población al cual está orientada la oferta académica (Amaya, 2005). En este orden de ideas, las variables que permiten formular las estrategias de diferenciación y enfoque, en torno al direccionamiento estratégico que tiene actualmente la universidad y el programa, surgen precisamente del análisis interno y externo de su estructura organizacional.

Finalmente teniendo en cuenta los resultados del análisis de los ambientes interno y externo de la Tecnología de Audio, junto con la información encontrada en la actividad preliminar de diagnóstico antes mencionada, se definió la estrategia maestra del programa con el fin de establecer los lineamientos básicos que moderarán los procesos y el desempeño futuro del mismo.

En este último paso se crearon en sí los planteamientos estratégicos del programa a través de tres aspectos centrales; la definición de la misión, la visión y los objetivos estratégicos de carácter académico y administrativo. 


\section{Misión}

El programa de Tecnología en Audio tiene como misión contribuir a la educación para todos a través de la modalidad abierta y a distancia, mediante la investigación, la acción pedagógica, la proyección social y las innovaciones metodológicas y didácticas, con la utilización de las tecnologías de la información y de las comunicaciones, para fomentar y acompañar el aprendizaje autónomo, generador de cultura y espíritu emprendedor que en el marco de la sociedad global y del conocimiento propicie el desarrollo económico, social y humano sostenible de las comunidades locales, regionales y globales con calidad, eficiencia y equidad social.

\section{Visión}

Se proyecta como un programa líder en educación abierta y a distancia a través de la metodología a distancia virtual de enseñanza y aprendizaje, reconocida a nivel nacional e internacional por la calidad innovadora y pertinencia de sus ofertas y servicios educativos y por su compromiso y aporte de su comunidad académica al desarrollo humano sostenible, de las comunidades locales y globales.

Para el establecimiento de los objetivos estratégicos de la Tecnología de Audio se diseñó una matriz DOFA que relacionó las fortalezas, debilidades, oportunidades y amenazas identificadas en el análisis de los entornos. Los resultados obtenidos fueron los siguientes:

\section{Objetivos Estratégicos Académicos:}

- Establecer proceso para la capacitación docente no sólo en materia de pedagogía y docencia universitaria, sino también para la especialización de cada uno de ellos en sus saberes específicos profesionales.

- Desarrollar procesos y espacios para la investigación con la finalidad de incrementar la calidad académica del programa.
- Adaptar la malla curricular y el plan de estudios del programa, para ajustarlo según las expectativas y necesidades de aprendizaje de los usuarios directos del servicio educativo.

- Ampliar el portafolio de productos del programa ofreciendo cursos de extensión, diplomados o abriendo espacios al público en general para el trabajo práctico en los laboratorios específicos de audio.

\section{Objetivos Estratégicos Administrativos:}

- Ampliar el número de laboratorios y de convenios con otras instituciones públicas y privadas en otras regiones del país diferente a Bogotá, para brindar soporte logístico y de infraestructura a los estudiantes que no se encuentran en el distrito capital.

- Fortalecer los vínculos entre la Universidad y las empresas. Establecer alianzas estratégicas entre la académica y los sectores productivos y sociales del país.

- Implementar el programa de Ingeniería de Audio y adaptarlo a las expectativas y necesidades sociales y de mercado que tengan los potenciales usuarios directos del servicio educativo.

- Establecer procesos de planificación para el diseño e implementación de planes de promoción ajustados que señalen los aspectos diferenciadores de la oferta educativa (mercadeo).

\section{Estado Actual del Programa}

En el marco del estudio realizado los resultados más significativos fueron los siguientes:

A nivel general se pudo inferir que la estructura organizacional de la Tecnología de Audio se encuentra relativamente débil. 
El análisis interno de los factores más determinantes del programa educativo en mención, puso en evidencia la necesidad de mejorar los factores estratégicos evaluados en la etapa de diagnóstico, en aspectos como la investigación, laboratorios, prácticas, convenidos, ajuste de contenidos, la promoción y la gestión.

Se identificaron las debilidades de la Tecnología de Audio que son el resultado en alto grado de múltiples fenómenos entre los que se destacan las continuas reformas estructurales y curriculares, el estado de crecimiento y maduración por el cual atraviesa el programa, al igual que la baja continuidad en los procesos académicos y de gestión hasta finales de 2010.

En menos de tres años se han ejecutado tres reformas que incluyen la modificación de la modalidad de estudio; pasó de ser tradicional a virtual e-learning, la trasformación de la malla curricular inicial; antes de 100 créditos ahora de 83, el cambio del docente a cargo del programa, junto con la reorientación de las diferentes líneas disciplinares que dan el enfoque especifico al mismo.

En cuanto al mercado, para la Tecnología de Audio el estar en la primera fase del ciclo de vida del producto, significa estar en un proceso de construcción integral del complejo organizacional que da soporte al mismo, es decir, el programa apenas está empezando a establecer, diseñar y optimizar el entramado de procesos necesarios para cumplir adecuadamente con la oferta educativa de formación tecnológica. Aspectos como el bajo volumen en ventas; matrículas, limitaciones en cuanto a cobertura, infraestructura, recursos económicos, canales de promoción, personal docente capacitado y material logístico y didáctico que soporten y fortalezcan el servicio ofrecido en términos de calidad, eficiencia y eficacia, son algunos de los elementos que enmarcan y ponen en evidencia la etapa introductoria de la oferta educativa.
Este período se caracteriza por la rentabilidad negativa, lo cual indica, que es completamente necesario realizar importantes esfuerzos en publicidad y distribución. Desde el punto de vista estratégico, el objetivo principal es expandir el mercado (Pride, 1996).

Teniendo en cuenta el ciclo de vida del producto y los resultados estadísticos obtenidos en el análisis de la encuesta aplicada, fue posible establecer el segmento de mercado sobre el cual se deben enfocar los esfuerzos de mercadeo en tres de los aspectos fundamentales de la mezcla de mercado, el producto, la plaza y la promoción, en función de las estrategias generales de diferenciación y de enfoque que se quieren implementar.

Por otro lado, la etapa introductoria del ciclo del producto se manifiesta también a través del comportamiento del número de estudiantes matriculados desde la primera promoción hasta la fecha en el programa académico. Para empezar, hasta el 2012 sólo se habían inscrito un total de 189 estudiantes (2008-2012) de los cuales un poco más de la mitad se retiraron en 2009 y 2010. Casi el $80 \%$ de los estudiantes retirados lo hicieron inmediatamente después de haber cursado un solo periodo académico en el programa. Las razones que se encontraron para que estos alumnos desistan de continuar con sus estudios fueron en primer lugar, que no se adaptaron a la modalidad académica planteada; e-learning, segundo porque no se sintieron a gusto con el plan curricular ofrecido y tercero porque el programa no les ofrecía una solución a sus necesidades de aprendizaje.

No obstante, el número de estudiantes inscritos cada año ha estado aumentando constantemente a una tasa variable.

El ingreso más significativo de estudiantes al programa se registró en el año 2009 y 2012 con una tasa de variación del 190\% y 31\% respectivamente. Estos cambios tan marcados fueron 
el resultado de la incorporación de iniciativas de difusión publicitaria de la Tecnología de Audio. En 2008 se repartieron por todos los Nodos Zonales de la UNAD en el país, publicidad impresa que promocionaba el programa de formación.

Para el 2011 se repartió nuevamente publicidad impresa y se inicio la implementación de algunas de las estrategias promocionales definidas en el plan estratégico de mercadeo online; apertura de espacios en redes sociales, publicidad en línea y publicación de contenidos sobre medios virtuales.

Estas dos actividades junto con la participación constante de la UNAD en las ferias de educación superior que se organizan en las principales ciudades del país, impulsaron la oferta del programa.

Por otro lado, el desarrollo de la encuesta permitió conocer claramente cuáles eran las expectativas de formación de los estudiantes del programa en relación a los contenidos temáticos y líneas de profundización que hacen parte del plan de estudios.

Los estudiantes que ingresan al programa Tecnología en Audio mostraron una clara inclinación hacia los campos de estudio relacionados con la ingeniería de sonido, la producción musical, audiovisual y la música.

\section{Autoevaluación Tecnología de Audio.}

El proceso de Autoevaluación de la Tecnología de Audio encontró en el estudio de mercado y en especial en la etapa inicial de diagnóstico, elementos de análisis muy importantes que contribuyeron en el desarrollo del Plan de Mejoramiento del programa para el periodo 2008 -2015.

Los resultados del estudio al igual que los indicadores cuantitativos y cualitativos subyacentes al mismo, fueron correlacionados con las ba- ses estadísticas oficiales de la universidad en relación al programa, con el fin de consolidar un trabajo de análisis, diagnóstico y planeación mucho más riguroso.

De este modo tras la finalización del ejercicio de Autoevaluación de la Tecnología de Audio, se evidenciaron las fortalezas, debilidades y las oportunidades de mejora de cada uno de los factores los 8 factores analizados en la misma, encontrando que se debía mejorar significativamente en aspectos como:

- Prácticas presenciales.

- Prácticas virtuales.

- Laboratorios.

- Investigación.

- Promoción.

- Convenios interinstitucionales.

- Rediseño curricular.

Si se comparan los resultados alcanzados en el proceso de Autoevaluación con los obtenidos en el estudio de mercado en su etapa previa de diagnostico e identificación de fortalezas, debilidades, amenazas y oportunidades del programa, se encuentra una amplia correlación entre los mismos, puesto que se desarrollaron aplicando metodologías similares, con elementos analíticos independientes y transversales entre el uno y el otro.

\section{Ingeniería de Audio.}

El trabajo de diagnostico de la Tecnología de Audio que incluyo el análisis de factores externos al programa importantes, permitió conocer algunos elementos relevante que se tuvieron en cuenta al momento de orientar el sentido formativo del programa profesional de pregrado, como por ejemplo la necesidad y expectativas del estudiante de encontrar cursos en el plan curricular, que abordaran principalmente temas en el campo de la producción musical y audiovisual. La investigación de mercado permitió caracterizar a la población objetivo a 
la cual estaría orientada la oferta académica, además de poner en evidencia las necesidades que tendría la misma en relación a sus expectativas de formación.

\section{Plan de Mercadeo}

Zapata (2007) menciona que:

Durante muchos años, las instituciones educativas crecieron gracias a la inercia propia de un mercado poco competitivo. Empero, la situación que deben encarar en la actualidad, tanto en Colombia como en el resto del mundo, es muy diferente. La fuerte e intensa competencia que deben enfrentar, las obliga a responder a una demanda exigente, con propuestas innovadoras, ajustadas a las necesidades del mercado al cual dirigen sus ofertas educativas (p.15).

En esta etapa se desarrollaron las siguientes actividades:

- Estudio del mercado y la competencia

- Definición de la estrategia global de mercadeo.

- Definición del posicionamiento y la mezcla de mercadeo.

El estudio del mercado y la competencia fue la etapa preliminar para la formulación del plan de mercadeo para la promoción de la Tecnología de Audio.

El estudio partió del análisis cuantitativo y cualitativo de las diferentes variables que hacen parte del mercado y la competencia directamente relacionados con la oferta académica, con el fin de establecer los segmentos de mercado potenciales y caracterizar la competencia directa del programa.

Ahora bien, para desarrollar y dar cumplimiento a los tres aspectos antes mencionados, se de- sarrolló un instrumento de recolección de datos que facilitó, tras su posterior revisión estadística y análisis, la identificación de algunas particularidades inherentes al conjunto de estudiantes activos y matriculados en el programa de Tecnología de Audio.

La encuesta tenía como objetivo encontrar datos que permitieran establecer el perfil de los estudiantes que ingresan a la Tecnología de Audio, para así determinar con mayor precisión las características generales que describen al estudiantado como un grupo especifico de personas con patrones de comportamiento similares. En ese orden de ideas se creó un formulario constituido por cinco secciones diferentes:

1. Información básica.

2. Información académica.

3. Información laboral.

4. Información de Tecnologías de la Información y las Comunicaciones.

De esta manera el segmento de mercado se definió teniendo en cuenta variables de carácter socioeconómico, geográficas y de conducta como se presenta enseguida.

Variables socioeconómicas: En este grupo se encontraron tres variables; sexo, rango de edad y educación, que permitieron definir características comunes dentro de la población encuestada. Los resultados fueron los siguientes:

Género: La promoción del programa estará orientada especialmente a personas del género masculino ya que es el mercado potencial con mayor prospectiva de crecimiento y consolidación.

Edad: El promedio de edad de los estudiantes que ingresan a la Tecnología en Audio es de 27 años y el mayor número de ellos están en el rango de edad comprendido entre los 19 y 28 años; aproximadamente el $71 \%$, por tal razón 
el segmento de edad al cual estará orientada la promoción del programa será el comprendido entre los 19 y 30 años de edad.

Educación: Los estudiantes que ingresan al programa Tecnología en Audio muestran una clara inclinación hacia los campos de estudio relacionados con la ingeniería de sonido, la producción musical y la música, además de señalar un interés, pero en menor significancia, por las líneas de formación relacionadas con la acústica y los sistemas de audio.

En este sentido, el plan de mercadeo estará orientado especialmente hacia los músicos y personas interesadas por la producción musical. En segundo orden se enfocará en las personas atraídas por la acústica y los sistemas de audio.

Variables geográficas: En este grupo se encontraron tres variables que ayudaron a definir características comunes dentro de la población encuestada; región, departamentos y zona territorial. Los resultados fueron los siguientes:

Regiones: Las zonas geográficas de donde provienen históricamente más del $80 \%$ de los estudiantes inscritos en el programa son la Región Andina y la Región del Caribe. Por tal razón las prácticas promocionales del programa se concentraran en dichas regiones.

Departamentos: Más del $80 \%$ de los estudiantes matriculados en la Tecnología de Audio vienen de algunos de los departamentos de la región Andina y del Caribe. Cundinamarca es el Departamento que aporta la mayor cantidad de estudiantes, seguido por los Departamentos del Atlántico, Boyacá, Cesar, Santander, Bolívar, la Guajira, Nariño, Antioquia y el Valle del Cauca.

Finalmente se genero la estrategia global de mercadeo enfocada especialmente en tres de los cuatro elementos que hacen parte de la mezcla de mercado, el producto, la plaza y la promoción, teniendo en cuenta que la dirección general del planteamiento estratégico apunta hacia la construcción de estrategias de diferenciación y alta segmentación, con la intención de incorporar o resaltar en el servicio, características tangibles o intangibles, que la demanda potencial de la oferta académica perciban como diferentes y que respondan a las necesidades y expectativas del segmento definido (Amaya, 2005).

\section{Consideraciones Finales}

Con la estructura y metodología de análisis aquí presentada para la creación del diagnóstico y el direccionamiento estratégico del programa en mención, se busca contribuir a la Universidad Nacional Abierta y a Distancia UNAD y en especial a la Escuela de Ciencias Básicas, Tecnología e Ingeniería (ECBTI), con planteamientos, elementos e instrumentos que puedan ser empelados en la construcción de un modelo de planeación prospectiva y estratégica formal, que sea capaz de adaptarse a las particularidades propias de cada programa de formación Tecnológica y Profesional de la Escuela, con el fin de fortalecer la estructura organizacional de la misma, mejorar en términos de calidad eficiencia y eficacia todos sus procesos, para así garantizar su crecimiento y permanencia a lo largo del tiempo.

En este sentido la discusión central se enfocaría en la determinación y construcción de los factores, metodologías de análisis, conceptos y demás elementos que integrarían el modelo de diagnóstico y de planeación estratégica que permitiría la revisión y determinación rigurosa del estado actual y la proyección futura de los diferentes programas que hacen parte de la Escuela. 
La planeación estratégica es el proceso que se establece con el propósito de desarrollar alternativas formales de acción, con el fin de mejorar el desempeño organizacional de las instituciones en el corto, mediano y largo plazo (López, 2005).

En este sentido es importante señalar que los procesos de planeación estratégica prospectiva si son importantes cuando su finalidad es el fortalecimiento de las estructuras y los procesos existentes en una organización en particular, puesto que ayudan a identificar; cuando están bien direccionados y su procesos de elaboración es riguroso, las debilidades, fortalezas, oportunidades y amenazas con las que se enfrenta el complejo organización, planteando la lineamientos fundamentales sobre los cuales orbitan las estrategias prospectivas de acción que se van a emplear para hacer frente a las amenazas del entorno.

Existen corrientes teóricas que argumentan que la planeación estratégica no tiene potencial para mejorar el desempeño de las organizaciones, puesto que generan burocracia, incremento en los costos e inflexibilidad en los procesos (López, 2005). En principio dicho argumento puede resultar verdadero, en el sentido de que se genera prácticas burocráticas a lo largo de todo el proceso de formulación e implementación, dando como resultado la formulación de diagnósticos y estrategias de acción sesgadas e inflexibles que muchas veces van en detrimento de la identidad y los objetivos organizacionales, pero esto ocurre, precisamente cuando el trabajo de planeación es centralizado y no participativo, cuando el acceso a la información es limitado, cuando los encargados de su realización toman decisiones en función de intereses particulares y cuando las bases teóricas y metodológicas aplicadas en la formulación e implementación del mismo no son las más adecuadas.
En contraste, los procesos de planeación estratégica que se desarrollan de manera participativa, con acceso libre a la información requerida, con mecanismos que regulen la intervención y los alcances de los aportes generados por los encargados de su formulación en función de intereses colectivas y no individuales, además de bases teóricas y metodológicas adecuadas, pueden generar resultados muy valiosos que contribuyan en la construcción de un plan de acción estratégico que realmente fortalezca la estructura y los procesos organizacionales.

Por otro lado y dejando a un lado la idea hasta ahora desarrollada, es importante establecer la discusión en relación a la viabilidad y pertinencia de la aplicación de iniciativas de mercadeo ajustadas que nazcan en el seno de cada uno de los programas académicos de la Universidad.

En el trabajo desarrollado en la Tecnología de Audio, se generaron elementos interesantes que deberían articularse a los procesos de mercadeo institucional que se llevan a cabo en la UNAD, como la formulación de investigaciones de mercado que permitan la identificación especifica de los usuarios potenciales de cada una de las ofertas académicas de la Universidad y, la implementación de prácticas de mercadeo diferentes a las ortodoxas Offline como aquellas que se hacen a través de medios virtuales.

De esta manera es importante generar discusión y conocimiento en relación a los dos aspectos antes mencionados, ya que es necesario generar en la Universidad, un círculo virtuoso de conocimiento que tenga como finalidad la articulación de modelos y prácticas académicas y administrativas, que impacten positivamente a la institución en el ejercicio de sus funciones educativas, sociales y de mercado. 


\section{Referencias}

Amaya, J. (2005). Gerencia, Planeación y Estrategia. Bucaramanga: Universidad Santo Tomás de Aquino.

Aranda, J. y Salgado, E. (2005). El Diseño Curricular y la Planeación Estratégica. Revista Innovación Educativa, 5, 25-35.

Beltrán, M. (2011). La Investigación sobre la Planeación Educativa. Revista Perfiles Educativos, 131: 3-6.

Fayol, H. (1916). Administración Gerencial y General. Paris: DunotEditeur.

Fred, R. (1989). La Gerencia Estratégica. Bogotá: LEGIS Fondo Editorial.

López, A. (2005). La Planeación Estratégica en la Pequeña y Mediana Empresa: una Revisión Bibliográfica. Revista EconoQuantum, 2: 141-164.

McGinn, N. y Warwick, D. (2006). La Planeación Educativa ¿Ciencia o Política? Revista Latinoamericana de Estudios Educativos. Vol. XXXVI, 1-2: 153-182.

Moya, R. (2008). Mercadeo Académico en las Instituciones de Educación Superior. Revista IES de Bogotá. Management, 12: 155-179.

Pride, W., Rosas, G y Jany, C. (1996). Marketing: Conceptos y Estrategias. México: McGraw-Hill.

Rodríguez, A., Ramírez, L y Moreno, D. (2002). Factores para la Permanencia de un Programa Educativo a Distancia Basado en Tecnologías de Información. México: Universidad Autónoma de Aguascalientes.
Serna, H., Salazar, J. y Salgado, J. (2009). Mercadeo Estratégico. Teoría - Metodología - Herramientas. Bogotá: 3D Editores.

Zapata, E. (2007). Mercadeo Educativo: Estrategias para Promover Instituciones y Programas, México: McGraw Hill.

CNA. (2006). Indicadores para la Autoevaluación con Fines de Acreditación de Programas de Pregrado en las Modalidades a Distancia y Virtual. Colombia: Concejo Nacional de Acreditación.

Ministerio de Tecnologías de la Información y las Comunicaciones. (2010). Informe Trimestral de Conectividad. Colombia: Ministerio de Tecnologías de la Información y las Comunicaciones.

Universidad Nacional Abierta y a Distancia-UNAD. (2009). Acuerdo número 001 de 2009, por el cual se modifica la estructura curricular del programa Tecnología en Audio de la Universidad Nacional Abierta y a Distancia UNAD y se define las condiciones para su oferta en la modalidad E-Learning. Bogotá, Colombia: Universidad Nacional Abierta y a Distancia.

Universidad Nacional Abierta y a Distancia-UNAD. (2006). Propuesta académica tecnología en audio oferta académica a distancia virtual. No. de resolución registro calificado: SNIES 52932. Bogotá, Colombia: Universidad Nacional Abierta y a Distancia.

Universidad Nacional Abierta y a Distancia-UNAD. (2005). Propuesta para la creación del programa Tecnología en Audio. Estándar de calidad no. 1 (justificación del programa decreto 2566 del 10 de septiembre de 2003). Bogotá, Colombia: Universidad Nacional Abierta y a Distancia. 